\title{
Erratum: Quantum simulation of long-range $X Y$ quantum spin glass with strong area-law violation using trapped ions [Phys. Rev. A 99, 052342 (2019)]
}

\author{
Nilanjan Roy, Auditya Sharma, and Rick Mukherjee
}

(Received 18 October 2019; published 13 November 2019)

DOI: 10.1103/PhysRevA.100.059902

An error was found in the code that generated the data presented in Figs. 2 and 3 of the published paper. The new corrected data are given below.

The corrected version of Fig. 2 pertains to the $X Y$ spin glass. There is a power-law violation of the area law of the entanglement entropy for small subsystem sizes, whereas for larger subsystem sizes, the power-law violation tends to disappear. The power-law violation of entanglement entropy persists until $\alpha=1$ beyond which $S^{A}$ vs $L$ plots do not show a smooth dependence on $L$ for which $\beta$ is difficult to extract by fitting. So we conclude that the power-law violation in quantum $X Y$ spin glass happens for the $\alpha \leqslant 1$ regime and the amount of power-law violation is comparatively less than what is found in the free fermionic model with random longrange hoppings.

The corrected version of Fig. 3 pertains to the $X Y$ disordered ferromagnet and antiferromagnet. The numbers have changed slightly now, but the conclusions from these figures remain the same as mentioned in the published paper.
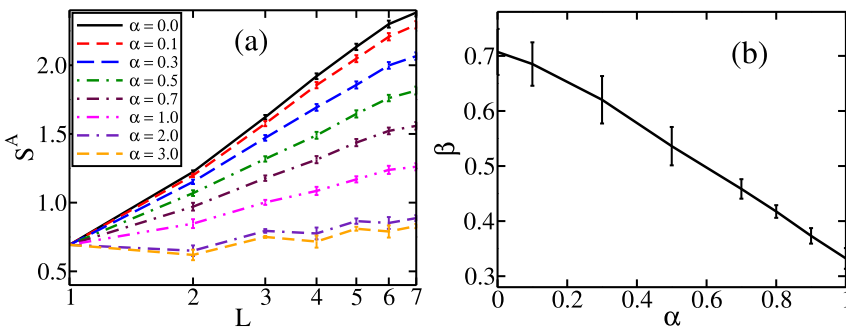

FIG. 2. Panels (a) and (b) depict the characteristics of the entanglement entropy for the one-dimensional quantum $X Y$ spin glass. For all the plots, we have taken 100 disorder realizations. (a) Scaling of the entanglement entropy $S^{A}$ with the subsystem size $L$ for increasing values of $\alpha$ in the one-dimensional long-range quantum $X Y$ spin glass of $N=16$ spins. The $x$ axis is on the logarithmic scale. (b) The exponent $\beta$ obtained from scaling of the entanglement entropy with the subsystem size $\left(S^{A} \propto L^{\beta}\right)$ as a function of $\alpha$ for system size $N=16$. For all the plots, disorder strength $V=1$.
The following points must be revised based on our new data analysis:

(1) In the abstract, the sentence "Strikingly this feature is found to be true even in the short-range regime, in sharp contrast to the spinless long-range disordered fermionic model" must be omitted.

(2) In the conclusion, a part of the sentence "which in contrast with the disordered long-range fermionic hopping model, extends into the short-range regime" must be cropped.

(3) From p. 3 left column of the published paper "Interestingly, when compared to the exponent...but to confirm this one would need to go to larger systems of the quantum spin glass system." should be removed.

In place of the above three points, the correct inference is as follows: We do see strong area-law violation for some values of $\alpha$ in both systems. However, the amount of power-law violation found in the quantum $X Y$ spin glass is comparatively less than what is found in the free fermionic model with random long-range hoppings.
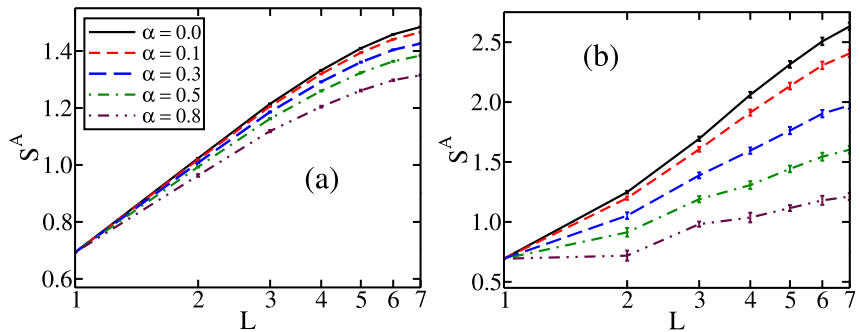

FIG. 3. (a) and (b) We compare entanglement properties of longrange spin chains where the $J$ 's of the couplings $J_{i j}$ are chosen from the distributions of negative random numbers $[-V, 0]$ and positive random numbers $[0, V]$, respectively, for $N=16$ spins with open boundaries. For all the plots, we have taken 100 disorder realizations. (a) Scaling of the entanglement entropy $S^{A}$ with the subsystem size $L$ (on the logarithmic scale) for increasing values of $\alpha$ for the spin chain with negative random couplings. (b) The entanglement entropy $S^{A}$ vs the subsystem size $L$ (on the logarithmic scale) for the same values of $\alpha$ for the spin chain with negative random couplings. For all the plots, $V=1$. 